PROCEEDINGS OF THE

AMERICAN MATHEMATICAL SOCIETY

Volume 126, Number 10, October 1998, Pages 2827-2834

S 0002-9939(98)04429-3

\title{
VECTOR BUNDLES WITH HOLOMORPHIC CONNECTION OVER A PROJECTIVE MANIFOLD WITH TANGENT BUNDLE OF NONNEGATIVE DEGREE
}

\author{
INDRANIL BISWAS
}

(Communicated by Ron Donagi)

\begin{abstract}
For a projective manifold whose tangent bundle is of nonnegative degree, a vector bundle on it with a holomorphic connection actually admits a compatible flat holomorphic connection, if the manifold satisfies certain conditions. The conditions in question are on the Harder-Narasimhan filtration of the tangent bundle, and on the Neron-Severi group.
\end{abstract}

\section{INTRODUCTION}

Let $E$ be a holomorphic vector bundle over a connected complex projective manifold $M$. Assume that $E$ admits a holomorphic connection. Then a natural question to ask is whether $E$ admits a flat holomorphic connection. Since all the rational Chern classes (of degree at least one) of a holomorphic vector bundle with a holomorphic connection vanish, there is no topological obstruction for the existence of a flat connection.

In this paper we consider this question for $M$ satisfying the condition that the degree of the tangent bundle $T_{M}$ is nonnegative with respect to some polarization on $M$.

Let

$$
0=V_{0} \subset V_{1} \subset V_{2} \subset \ldots \subset V_{l} \subset V_{l+1}=T_{M}
$$

be the Harder-Narasimhan filtration of the tangent bundle $T_{M}$ with respect to a polarization $L$ on $M$.

In Theorem 2.4 we prove the following (degree of a coherent sheaf on $M$ is computed using $L)$ :

Theorem A. Assume that the degree of the tangent bundle $\operatorname{deg} T_{M} \geq 0$. Let $E$ be a holomorphic vector bundle on $M$ equipped with a holomorphic connection.

(1) If $\operatorname{deg}\left(T_{M} / V_{l}\right) \geq 0$ then the holomorphic vector bundle $E$ admits a compatible flat connection. (This inequality condition is satisfied if, for example, $T_{M}$ is semistable, since $\operatorname{deg} T_{M} \geq 0$.)

Received by the editors February 24, 1997.

1991 Mathematics Subject Classification. Primary 14F05, 32L10, 53C07.

Key words and phrases. Holomorphic connection, flat connection, Harder-Narasimhan filtration. 
(2) Consider the case where $T_{M}$ is not semistable. Assume that the maximal semistable subsheaf of $T_{M}$, namely $V_{1}$, is locally free. If the rank of the NeronSeveri group, $N S(M)$, of $M$ is 1 , i.e.,

$$
H^{1,1}(M) \cap H^{2}(M, \mathbb{Q})=\mathbb{Q},
$$

then $E$ admits a compatible flat connection.

Under the assumptions either in part (1) or in part (2) of Theorem A, the vector bundle $E$ turns out to be semistable with respect to $L$ [Remark 2.12].

Generalizing the above question one may ask whether a holomorphic fiber bundle admitting a holomorphic connection actually admits a flat holomorphic connection. S. Murakami produced an example of a holomorphic fiber bundle over an abelian variety, with an abelian variety as fiber, such that the fiber bundle admits a holomorphic connection, but it does not admit any flat holomorphic connection [M1], [M2], [M3]. However part (1) of Theorem A implies that any holomorphic vector bundle over a projective manifold with trivial canonical line bundle, which admits a holomorphic connection, actually admits a flat holomorphic connection. Indeed, by a theorem of Yau [Ya] the tangent bundle of such a variety is semistable.

On the other hand, using a method of [Bi2], Theorem A can easily be generalized to principal $G$-bundles, where the structure group $G$ is a connected affine algebraic reductive group over $\mathbb{C}$. The example of Murakami shows that it is essential for $G$ to be noncompact.

\section{Criteria For the EXistence of a flat CONNECtion}

Let $M / \mathbb{C}$ be a connected smooth projective variety of complex dimension $d$. We will denote by $T_{M}$ (resp. $\Omega_{M}^{1}$ ) the holomorphic tangent bundle (resp. cotangent bundle) of $M$.

For a holomorphic vector bundle $V$, the corresponding coherent analytic sheaf given by its local holomorphic sections will also be denoted by $V$. The basic facts about holomorphic structures used here can be found in [Ko].

A holomorphic connection on a holomorphic vector bundle $E$ over $M$ is a first order differential operator

$$
D: E \longrightarrow \Omega_{M}^{1} \otimes E
$$

satisfying the following Leibniz condition:

$$
D(f s)=f D(s)+d f \otimes s
$$

where $f$ is a local holomorphic function on $M$ and $s$ is a local holomorphic section of $E$. Extend $D$ as a first order operator

$$
D: \Omega_{M}^{p, q} \otimes E \longrightarrow \Omega_{M}^{p+1, q} \otimes E
$$

using the Leibniz rule. The curvature of $D$ is defined to be

$$
D^{2}:=D \circ D
$$

which is a holomorphic section of $\Omega_{M}^{2} \otimes$ End $E\left(\Omega_{M}^{k}:=\bigwedge^{k} \Omega_{M}^{1}\right)$. The notion of a holomorphic connection was introduced by M. Atiyah [At].

If $\bar{\partial}_{E}: E \longrightarrow \Omega_{M}^{0,1} \otimes E$ denotes the first order differential operator defining the holomorphic structure on $E$, then the operator

$$
D+\bar{\partial}_{E}
$$


is a connection on $E$ in the usual sense. Moreover, the curvature of this connection is $D^{2}$; in particular, it is a holomorphic section of $\Omega_{M}^{2} \otimes$ End $E$. Conversely, the $(1,0)$ part of a connection on $E$, such that the $(0,1)$ part of it is $\bar{\partial}_{E}$ and its curvature is a holomorphic section of $\Omega_{M}^{2} \otimes$ End $E$, is actually a holomorphic connection.

In particular, if $\nabla$ is a flat connection on a $C^{\infty}$ complex vector bundle $M$, then the $(0,1)$ part of the connection operator defines a holomorphic structure on $E$ and the $(1,0)$ part defines a holomorphic connection.

Let $L$ be a polarization on $M$, or equivalently, $L$ is an ample line bundle on $M$. For a coherent sheaf $F$ on $M$, the degree of $F$, denoted by $\operatorname{deg} F$, is defined as follows $\left(d=\operatorname{dim}_{\mathbb{C}} M\right)$ :

$$
\operatorname{deg} F \quad:=\quad \int_{M} c_{1}(F) \cup c_{1}(L)^{d-1} .
$$

A torsion-free coherent sheaf $F$ is called semistable if for every (nonzero) coherent subsheaf $V \subset F$, the following inequality holds:

$$
\frac{\operatorname{rank} V}{\operatorname{deg} V} \leq \frac{\operatorname{rank} F}{\operatorname{deg} F}
$$

Moreover, if the strict inequality holds for every proper coherent subsheaf $V$ with $F / V$ torsion-free, then $F$ is called stable.

The quotient $\operatorname{rank} F / \operatorname{deg} F$ is called the slope of $F$ and is usually denoted by $\mu(F)$.

Any torsion-free coherent sheaf $F$ admits a unique filtration by coherent subsheaves, known as the Harder-Narasimhan filtration, of the following type ([Ko], page 174, Theorem 7.15):

$$
0=F_{0} \subset F_{1} \subset F_{2} \subset \ldots \subset F_{k} \subset F_{k+1}=F
$$

where $F_{1}$ is the maximal semistable subsheaf of $F$. The Harder-Narasimhan filtration is determined by the property that $F_{i+1} / F_{i}$ is the maximal semistable subsheaf of $F / F_{i}$. This implies that $\mu\left(F_{i+1} / F_{i}\right)<\mu\left(F_{i} / F_{i-1}\right)$.

Let

$$
0=V_{0} \subset V_{1} \subset V_{2} \subset \ldots \subset V_{l} \subset V_{l+1}=T_{M}
$$

be the Harder-Narasimhan filtration of the tangent bundle $T_{M}$.

A flat connection on a holomorphic vector bundle $E$ on $M$ is said to be compatible if the $(0,1)$ part of the connection is $\bar{\partial}_{E}$ (equivalently, (local) flat sections are holomorphic sections). A compatible flat connection is same as a flat holomorphic connection.

Theorem 2.4. Assume that the degree of the tangent bundle deg $T_{M} \geq 0$. Let $E$ be a holomorphic vector bundle on $M$ equipped with a holomorphic connection.

(1) If $\operatorname{deg}\left(T_{M} / V_{l}\right) \geq 0$ then the holomorphic vector bundle $E$ admits a compatible flat connection. (This inequality condition is satisfied if, for example, $T_{M}$ is semistable since $\operatorname{deg} T_{M} \geq 0$.)

(2) Consider the case where $T_{M}$ is not semistable. Assume that the maximal semistable subsheaf of $T_{M}$, namely $V_{1}$, is locally free. If the rank of the NeronSeveri group, $N S(M)$, of $M$ is 1 , i.e.,

$$
H^{1,1}(M) \cap H^{2}(M, \mathbb{Q})=\mathbb{Q},
$$

then $E$ admits a compatible flat connection. 
Proof. Assume that $\operatorname{deg}\left(T_{M} / V_{l}\right) \geq 0$. Then from Lemma 2.1 of [Bi2] (also Remark 3.7(ii) of [Bi1]) we know that the vector bundle $E$ is semistable. To be self-contained as much as possible we will quickly recall the proof of the semistability of $E$. Since $E$ admits a holomorphic connection, Theorem 4 (page 192) of [At] says that all the (rational) Chern classes, $c_{k}(E)$, where $k \geq 1$, of $E$ vanish. In particular $\operatorname{deg} E=0$. Let $W$ be the maximal semistable subsheaf of $E$. The key observation is that $W$ is left invariant by the holomorphic connection operator $D$ on $E$. Indeed, the homomorphism

$$
W \longrightarrow \Omega_{M}^{1} \otimes \frac{E}{W}
$$

induced by $D$ is $\mathcal{O}_{M}$-linear (a simple consequence of the Leibniz identity (2.2)). The Harder-Narasimhan filtration of a tensor product is simply the tensor product of the corresponding Harder-Narasimhan filtrations. Applying this to $\Omega_{M}^{1} \otimes(E / W)$, since the degree of any subsheaf of $\Omega_{M}^{1}$ is nonpositive (this is equivalent to the assertion that the degree of a quotient sheaf of $T_{M}$ is nonnegative, which, in turn, is warranted by the assumption that $\left.\operatorname{deg}\left(T_{M} / V_{l}\right) \geq 0\right)$, the slope of the maximal semistable subsheaf of $\Omega_{M}^{1} \otimes(E / W)$ is less than or equal to $\mu(E / W)$. Finally from the general properties of Harder-Narasimhan filtrations we have $\mu(W)>\mu(E / W)$. If the image of the homomorphism in (2.5) is nonzero then the slope of the image is simultaneously at least $\mu(W)$ (recall that $W$ is semistable) and as well as it is at most the slope of the maximal semistable subsheaf of $\Omega_{M}^{1} \otimes(E / W)$. This contradicts the earlier observation that the slope of the maximal semistable subsheaf of $\Omega_{M}^{1} \otimes(E / W)$ is strictly less than $\mu(W)$. Thus the homomorphism in (2.5) must be the zero homomorphism. In other words, $W$ has an induced holomorphic connection. This implies that $W$ is locally free of degree zero. So $W$ cannot be a proper subsheaf of $E$. In other words, $E$ must be semistable.

Since $E$ is semistable with vanishing first and second Chern classes, the Corollary 3.10 (page 40 ) of [Si] implies that $E$ admits a flat connection compatible with its holomorphic structure.

To prove part (2) of Theorem 2.4 we assume that $T_{M}$ is not semistable. The maximal semistable subsheaf of $T_{M}$, namely $V_{1}$ (in (2.3)), is assumed to be locally free.

Our first step will be to prove that $V_{1}$ is closed under the Lie bracket operation on $T_{M}$. Towards this goal consider the homomorphism

$$
\Gamma: V_{1} \otimes V_{1} \longrightarrow \frac{T}{V_{1}}
$$

defined by composing the Lie bracket operation with the natural projection of $T_{M}$ onto $T_{M} / V_{1}$. Since the Lie bracket satisfies the Leibniz identity, namely

$$
[f v, w]=f[v, w]-\langle d f, w\rangle v,
$$

where $\langle-,-\rangle$ denotes the obvious contraction, the map $\Gamma$ is actually $\mathcal{O}_{M}$-linear, i.e., $\Gamma$ is a homomorphism of vector bundles.

Now we are given that $\mu\left(V_{1}\right)>\mu\left(T_{M}\right) \geq 0$. So

$$
\mu\left(V_{1} \otimes V_{1}\right)=2 \mu\left(V_{1}\right)>\mu\left(V_{1}\right)>\mu\left(V_{2} / V_{1}\right),
$$

the last inequality being a general property of Harder-Narasimhan filtrations. The image of the homomorphism $\Gamma$ is simultaneously a quotient of $V_{1} \otimes V_{1}$ as well as a 
subsheaf of $T_{M} / V_{1}$. But $V_{2} / V_{1}$, by definition, is the maximal semistable subsheaf of $T_{M} / V_{1}$. So if $\Gamma \neq 0$ then

$$
\mu\left(V_{1} \otimes V_{1}\right) \leq \mu(\text { image } \Gamma) \leq \mu\left(V_{2} / V_{1}\right) .
$$

The first inequality is a consequence of the fact that $V_{1} \otimes V_{1}$ is semistable. (A tensor product of semistable vector bundles is again semistable [MR], Remark 6.6 (iii).) This contradicts the inequality (2.7) unless image $\Gamma=0$. But $\Gamma=0$ is equivalent to $V_{1}$ being closed under the Lie bracket operation. In other words, $V_{1}$ is a nonsingular holomorphic foliation on $M$.

If $E$ is semistable we may complete the proof of Theorem 2.4 by repeating the use of the Corollary 3.10 of [Si] as done in the proof of part (1) of Theorem 2.4. So we may, and we will, assume that $E$ is not semistable. Let

$$
0=W_{0} \subset W_{1} \subset W_{2} \subset \ldots \subset W_{m} \subset W_{m+1}=E
$$

be the Harder-Narasimhan filtration of $E$.

Our next step will be to show that the sheaf $W_{1}$ has an induced holomorphic partial connection along the foliation $V_{1}$. In other words, we want to show that the operator $D$ in (2.1) induces an operator

$$
D^{\prime}: W_{1} \longrightarrow V_{1}^{*} \otimes W_{1}
$$

which satisfies the Leibniz condition (2.2); $d f$ in (2.2) is realized as a section of $V_{1}^{*}$ in (2.8) by using the natural projection of $\Omega_{M}^{1}$ onto $V_{1}^{*}$. The notion of a partial connection was introduced by R. Bott.

To construct $D^{\prime}$ first note that, by projecting $\Omega_{M}^{1}$ onto $V_{1}^{*}$, the operator $D$ in (2.1) induces an operator

$$
D_{1}: W_{1} \longrightarrow V_{1}^{*} \otimes E .
$$

Now projecting $E$ onto $E / W_{1}$, the operator $D_{1}$ in (2.9) induces an operator

$$
D_{2}: W_{1} \longrightarrow V_{1}^{*} \otimes \frac{E}{W_{1}} \text {. }
$$

The Leibniz identity (2.2) implies that $D_{2}$ is $\mathcal{O}_{M}$-linear; i.e., the order of the differential operator $D_{2}$ is zero. In other words, $D_{2}$ is a homomorphism of vector bundles.

We will show that $D_{2}=0$ by following the steps of the argument for $\Gamma=0$ (in (2.6)).

If $D_{2} \neq 0$ then $\mu$ (image $\left.\left(D_{2}\right)\right) \geq \mu\left(W_{1}\right)$, since image $\left(D_{2}\right)$ is a quotient of the semistable sheaf $W_{1}$. On the other hand, since

$$
\text { image }\left(D_{2}\right) \subseteq V_{1}^{*} \otimes \frac{E}{W_{1}},
$$

we conclude that the slope of image $\left(D_{2}\right)$ is at most the slope of the maximal semistable subsheaf of $V_{1}^{*} \otimes\left(E / W_{1}\right)$.

Thus if $D_{2} \neq 0$, then $\mu\left(W_{1}\right)$ is less than or equal to the slope of the maximal semistable subsheaf of $V_{1}^{*} \otimes\left(E / W_{1}\right)$.

On the other hand, since $V_{1}^{*}$ is semistable with strictly negative slope, the slope of the maximal semistable subsheaf of $V_{1}^{*} \otimes\left(E / W_{1}\right)$ is strictly less than the slope of the maximal semistable subsheaf of $E / W_{1}$ - which in turn is strictly less than the slope of $W_{1}$. Thus the slope of the maximal semistable subsheaf of $V_{1}^{*} \otimes\left(E / W_{1}\right)$ is strictly less than $\mu\left(W_{1}\right)$. This contradicts the inequality obtained in the previous paragraph. So we have $D_{2}=0$. 
Since $D_{2}=0$, the differential operator $D_{1}$ in (2.9) induces a first order differential operator $D^{\prime}$ as in (2.8). Clearly $D^{\prime}$ satisfies the Leibniz identity, as $D$ satisfies it.

The operator $D^{\prime}$ maps (local) holomorphic sections of $W_{1}$ to holomorphic sections of $V_{1}^{*} \otimes W_{1}$. So $D^{\prime}$ is a partial connection on $W_{1}$ along $V_{1} \oplus T_{M}^{0,1}$ in the sense of [BB] (Sections 2 and 3); $T_{M}^{0,1}$ is the anti-holomorphic tangent bundle.

However, unfortunately, $W_{1}$ is not necessarily locally free. (A coherent sheaf equipped with a holomorphic connection must be locally free, but $D^{\prime}$ is only a partial connection.) To circumvent the problems caused by such a possibility of not being locally free, we will consider the determinant line bundle

$$
d\left(W_{1}\right) \quad:=\operatorname{det} W_{1}=\bigwedge^{r} W_{1}
$$

where $r$ is the rank of $W_{1}$. The details of the construction of the determinant bundle of a torsion-free coherent sheaf can be found in Chapter $5, \S 6$ of [Ko]. We note that the determinant bundle of a torsion-free sheaf is locally free of rank one, i.e., it is a line bundle.

The partial connection $D^{\prime}$ induces a partial connection on $d\left(W_{1}\right)$, which we will also denote by $D^{\prime}$. More precisely, for a local section of $d\left(W_{1}\right)$

$$
s:=s_{1} \wedge s_{2} \wedge \ldots \wedge s_{r} \in \Gamma\left(U, d\left(W_{1}\right)\right)
$$

the action of $D^{\prime}$ on it is defined as follows:

$$
D^{\prime}(s) \quad:=\sum_{j=1}^{r} s_{1} \wedge \ldots \wedge D^{\prime}\left(s_{j}\right) \wedge \ldots \wedge s_{r} .
$$

It is straight-forward to check that the operator $D^{\prime}$ defined above satisfies the Leibniz identity. Thus $D^{\prime}$ is a partial holomorphic connection on $d\left(W_{1}\right)$ along $V_{1}$.

We may extend the partial connection $D^{\prime}$ to an actual connection on $d\left(W_{1}\right)$ following $[\mathrm{BB}]$. Fix a Kähler metric, say $H$, on $M$. Let $\nabla^{\prime}$ be a hermitian connection on $d\left(W_{1}\right)$; the $(0,1)$ part of $\nabla^{\prime}$ is assumed to be $\bar{\partial}_{d\left(W_{1}\right)}$. For any $v \in T_{M}^{1,0}$ let $v=v_{1} \oplus v_{2}$ be the decomposition as $T_{M}^{1,0}=V_{1} \oplus V_{1}^{\perp}$ using the metric $H$. For $v^{\prime} \in T_{M}^{0,1}$ and a smooth section $\phi$ of $d\left(W_{1}\right)$ define:

$$
\nabla_{v \oplus v^{\prime}} \phi \quad:=\left\langle D^{\prime} \phi, v_{1}\right\rangle+\nabla_{v_{2}}^{\prime} \phi+\left\langle\bar{\partial}_{d\left(W_{1}\right)} \phi, v^{\prime}\right\rangle .
$$

Clearly $\nabla$ is a connection in the usual sense whose $(0,1)$ part coincides with $\bar{\partial}_{d\left(W_{1}\right)}$, and it is an extension of the partial connection $D^{\prime}$.

Let

$$
\mathcal{I} \subseteq \Omega_{M}^{1,1} \oplus \Omega_{M}^{0,2}
$$

be the degree 2 component of the ideal, in the exterior algebra $\wedge\left(\Omega_{M}^{1,0} \oplus \Omega_{M}^{0,1}\right)$, generated by the subspace of $\Omega_{M}^{0,1}$ that annihilates $\overline{V_{1}}$.

The following simple lemma will be useful:

Lemma 2.10. The curvature $\nabla^{2}$, which is a smooth 2 -form on $M$, is actually a section of $\mathcal{I} \oplus \Omega_{M}^{2,0}$.

The proof of Lemma 2.10 is a simple computation. It is actually a straightforward extension of (3.33), page 295 of [BB] to partial holomorphic connections (extension from partial flat connections). All we need to observe is that the cur- 
vature of $\nabla^{\prime}$ is of type $(1,1)$ (since $\nabla^{\prime}$ is assumed to be hermitian) and that the curvature of the partial connection $D^{\prime}$ is a holomorphic section of $\bigwedge^{2} V_{1}^{*}$. Since the restriction of $\nabla$ to a leaf of the foliation $V_{1}$ coincides with $D^{\prime}+\bar{\partial}_{d\left(W_{1}\right)}$, the restriction of $\nabla^{2}$ to a leaf is a section of $\bigwedge^{2} V_{1}^{*}$. It is easy to see that this implies Lemma 2.10.

Continuing with the proof of Theorem 2.4, our next step will be to establish a lemma on vanishing of characteristic classes of $d\left(W_{1}\right)$, analogous to the Proposition (3.27), page 295 , of $[\mathrm{BB}]$.

Lemma 2.11. Let $q$ be an integer with $q>\operatorname{dim} M-\operatorname{dim} V_{1}$. Then $c_{1}\left(d\left(W_{1}\right)\right)^{q}=0$.

Proof of Lemma 2.11. The characteristic class $c_{1}\left(d\left(W_{1}\right)\right)^{q} \in H^{q, q}(M)$, and it is represented by the differential form $\left(\nabla^{2} / 2 \pi \sqrt{-1}\right)^{q}$. Since the space of forms on $M$ admits Hodge decomposition, to prove Lemma 2.11 it is enough to show that the differential form $\left(\nabla^{2}\right)^{q}$ is a section of the vector bundle

$$
\bigoplus_{j>q} \Omega_{M}^{j, 2 q-j}
$$

But Lemma 2.10 implies that $\left(\nabla^{2}\right)^{q}$ is indeed of the above type. To see this first note that by Lemma 2.10 , both the $(1,1)$ and the $(0,2)$ part of $\nabla^{2}$ is contained in the ideal generated by the subspace of $\Omega_{M}^{0,1}$ that annihilates $\overline{V_{1}}$. But the dimension of this annihilator is $\operatorname{dim} M-\operatorname{dim} V_{1}$. So the component of $\left(\nabla^{2}\right)^{q}$ in

$$
\bigoplus_{j \leq q} \Omega_{M}^{j, 2 q-j}
$$

vanishes identically. This completes the proof of the lemma.

To complete the proof of Theorem 2.4 we first note that the given condition that the rank of the Neron-Severi group, $N S(M)$, is 1 implies that if $(\omega)^{j}=0$, where $\omega \in N S(M) \otimes_{\mathbb{Z}} \mathbb{Q}\left(=H^{2}(M, \mathbb{Q}) \cap H^{1,1}(M)\right)$ and $1 \leq j \leq \operatorname{dim}_{\mathbb{C}} M$, then $\omega=0$. This is simply because $\omega$ is a (possibly zero) rational multiple of the hyperplane class, and the $j$-th power of the hyperplane class is nonzero. Substituting $c_{1}\left(d\left(W_{1}\right)\right)$ for $\omega$ and using Lemma 2.11 we get that $c_{1}\left(d\left(W_{1}\right)\right)=0$. Thus we have

$$
\operatorname{deg} W_{1}=\operatorname{deg} d\left(W_{1}\right)=0 .
$$

But $W_{1}$ is the maximal semistable subsheaf of $E$ and $\operatorname{deg} E=0$. This contradicts the assumptions that $E$ is not semistable and that $W_{1}$ is the maximal semistable subsheaf of $E$. We already noted that if $E$ is semistable then the Corollary 3.10 (page 40) of $[\mathrm{Si}]$ completes the proof of the theorem. This completes the proof of Theorem 2.4.

Remark 2.12. The proof of Theorem 2.4 shows that under the assumptions in either part 1 or part 2 of the statement of Theorem 2.4, the vector bundle $E$ is actually semistable.

\section{ACKNOWLEDGMENTS}

The author is thankful to C. Simpson and T. Pantev for explaining some results of [Si] used here. 


\section{REFERENCES}

[At] M.F. Atiyah : Complex analytic connections in fibre bundles. Transactions of the Amer. Math. Soc. 85 (1957), 181-207. MR 19:172c

[BB] P. Baum and R. Bott : Singularities of holomorphic foliations. Jour. Diff. Geom. 7 (1972), 279-342. MR 51:14092

[Bi1] I. Biswas : On Harder-Narasimhan filtration of the tangent bundle. Comm. Anal. Geom. 3 (1995), 1-10. MR 97a:32031

[Bi2] I. Biswas : Principal bundles admitting a holomorphic connection. Int. Jour. Math. 7 (1996), 433-440. MR 97f:32043

[Ko] S. Kobayashi : Differential Geometry of Complex Vector Bundles. Publications of the Math. Society of Japan 15. Iwanami Shoten Publishers and Princeton University Press (1987). MR 89e:53100

[M1] S. Murakami : Sur certains espaces fibrés principaux admettant des connexions holomorphes. Osaka Math. Jour. 11 (1959), 43-62. MR 22:958

[M2] S. Murakami : Sur certains espaces fibrés principaux holomorphes dont le groupe est abélien connexe. Osaka Math. Jour. 13 (1961), 143-167. MR 26:788

[M3] S. Murakami : Harmonic connections and their applications. SEA Bull. Math. Special Issue (1993), 101-103. MR 94k:53042

[MR] V. Mehta and A. Ramanathan : Semistable sheaves on projective varieties and their restriction to curves. Math. Ann. 258 (1982), 213-224. MR 83f: 14013

[Si] C. Simpson : Higgs bundles and local systems. Pub. Math. IHES 75 (1992), 5-95. MR 94d:32027

[Ya] S. T. Yau : On Calabi's conjecture and some new results in algebraic geometry. Proc. Nat. Sci. U.S.A. 74 (1977), 1798-1799.

School of Mathematics, Tata Institute of Fundamental Research, Homi Bhabha ROAD, BOMBAY 400005, INDIA

E-mail address: indranil@math.tifr.res.in 